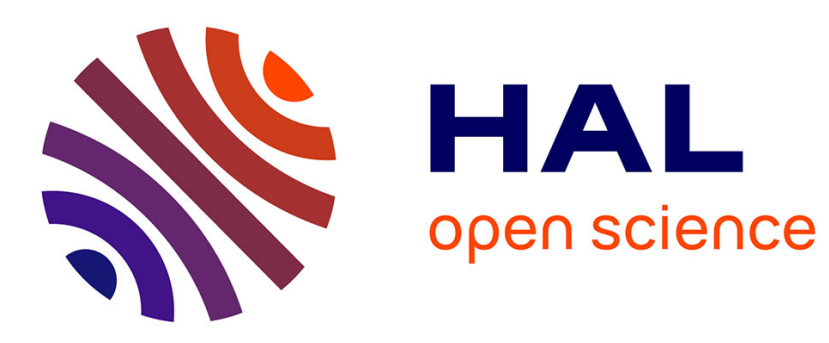

\title{
Identité professionnelle des enseignants : entre singularité des parcours et modes d'ajustement aux changements institutionnels
}

Thérèse Roux-Perez

\section{- To cite this version:}

Thérèse Roux-Perez. Identité professionnelle des enseignants : entre singularité des parcours et modes d'ajustement aux changements institutionnels. Savoirs: Revue internationale de recherches en éducation et formation des adultes, 2006, 11 (2), pp.107-123. 10.3917/savo.011.0107 . hal-01716201

\section{HAL Id: hal-01716201 \\ https://hal.science/hal-01716201}

Submitted on 23 Feb 2018

HAL is a multi-disciplinary open access archive for the deposit and dissemination of scientific research documents, whether they are published or not. The documents may come from teaching and research institutions in France or abroad, or from public or private research centers.
L'archive ouverte pluridisciplinaire HAL, est destinée au dépôt et à la diffusion de documents scientifiques de niveau recherche, publiés ou non, émanant des établissements d'enseignement et de recherche français ou étrangers, des laboratoires publics ou privés. 


\section{ROUX-PEREZ Thérèse \\ IUFM des Pays de la Loire \\ Laboratoire du CREN (Centre de Recherches en Education de Nantes)}

\section{Identité professionnelle des enseignants : entre singularité des parcours et modes d'ajustement aux changements institutionnels}

\section{Revue SAVOIRS n ${ }^{\circ} 11,2006$}

\section{Résumé}

Durant une carrière, certains changements institutionnels sont vécus par les acteurs comme problématiques et transforment leur relation au métier. L'article se donne pour objet de comprendre, à partir d'une étude de cas en Education Physique et Sportive (EPS), la manière dont les enseignants donnent sens à leur parcours professionnel, se positionnent par rapport aux recommandations officielles et s'investissent dans leur contexte de travail. Les résultats mettent en relief le rapport entre histoire personnelle et perception de l'évolution disciplinaire. Ils soulignent la quête de reconnaissance dans ou à l'extérieur du système scolaire. Enfin, ils montrent dans quelle mesure l'enseignant construit un monde cohérent, en gérant de multiples tensions. L'orientation des actions et les formes d'engagement choisies sont justifiées par des représentations et des valeurs. Celles-ci, fondatrices d'une identité professionnelle, peuvent éclairer les problématiques liées à la formation des enseignants.

\section{Mots clés}

Identité professionnelle, carrière, représentations, rapport au métier, formation 


\section{Identité professionnelle des enseignants : entre singularité des parcours et modes d'ajustement aux changements institutionnels.}

\section{Introduction}

Les mutations profondes de la société depuis une trentaine d'années et ses effets sur l'école ont affecté l'identité professionnelle des enseignants. La remise en cause des normes, des valeurs et des modèles de conduite a rendu plus complexes les processus d'identification de ces acteurs à l'institution scolaire (Dubet, 2002). Notre recherche s'intéresse à la question des parcours biographiques et des stratégies identitaires. Elle permet de comprendre dans quelle mesure les enseignants d'Education Physique et Sportive (EPS) donnent sens à leur parcours professionnel, se positionnent par rapport aux recommandations officielles et s'investissent dans leur contexte de travail. Elle s'attache particulièrement à repérer ce qui dans la formation initiale a pu fonder une identité professionnelle et rendre plus ou moins difficile, au cours de la carrière, le rapport à l'évolution disciplinaire. Cette contribution se donne pour objet de présenter le parcours d'un enseignant d'EPS caractéristique d'un positionnement problématique vis-à-vis des changements institutionnels dans sa discipline.

\subsection{L'EPS : une discipline en évolution}

Dans les années 1980, la volonté institutionnelle de placer l'élève au centre des préoccupations éducatives a visé la transformation des pratiques professionnelles en EPS : construction de projets pédagogiques, traitement didactique des Activités Physiques Sportives et Artistiques (APSA), réflexion sur les contenus à enseigner et sur les modalités d'évaluation, mise en place de procédures pédagogiques nouvelles (différenciation, évaluation formative, pédagogie du contrat, mise en projet de l'élève, etc.). Elle s'est accompagnée d'une offre de formation continue diversifiée. Pourtant, cette évolution a été vécue de façon très différente par les enseignants d'EPS : certains se sont inscrits dans la dynamique alors que d'autres, se sentant en décalage avec les compétences professionnelles nouvelles exigées par l'institution, ont eu tendance à résister à ce changement en estimant que l'EPS était en train de «perdre son identité ». Ces différences d'interprétation ont engendré des prises de positions relativement contrastées au sein du groupe professionnel (Roux-Perez, 2003a).

\subsection{Stratégies d'adaptation des acteurs aux évolutions professionnelles}

D'une manière générale, l'identité s'inscrit dans une perspective dynamique et intègre les différentes expériences de l'individu tout au long de la vie. Les multiples interactions entre le sujet et le monde qui l'environne participent à l'élaboration d'une identité à la fois multidimensionnelle et structurée en un tout plus ou moins cohérent et fonctionnel. Ainsi, malgré les évolutions auxquelles il est confronté, chacun garde conscience de son unité et de sa continuité, de même qu'il attend d'être reconnu par les autres comme quelqu'un de singulier, pouvant agir sur le choix de ses groupes d'appartenance et de référence (Merton, 1957).

Lorsque cet ensemble d'éléments n'est plus réuni, l'individu développe des stratégies identitaires (Camilleri et al , 1990), envisagées comme des marges de manœuvre face aux clivages internes et aux contradictions avec l'environnement. Ces stratégies sont négociées selon deux processus : un processus d'appartenance utilisé pour résoudre d'éventuels conflits identitaires ; un processus de différenciation apparaissant lorsque la singularité ou la spécificité de chacun se trouve menacée. 
ROUX-PEREZ.T, MCF, IUFM des Pays de la Loire, Laboratoire du CREN, Nantes, Identité professionnelle des enseignants : entre singularité des parcours et modes d'ajustement aux changements institutionnels. Revue Savoirs, 2006, n¹1, pp. 107-123.

Dans le cadre professionnel, les distorsions entre expériences passées et situations présentes amènent l'individu à développer une pluralité de logiques d'action fondant une mise en cohérence entre soi, les autres et le contexte de travail (Derouet, 1992 ; Dubar, 2000 ; Lahire, 1998). L'étude du cas que nous présentons dans cet article rend compte des réajustements à l'œuvre pour construire une cohérence et conserver un équilibre relatif dans l'exercice du métier.

\subsection{Identité professionnelle et carrière des enseignants}

En tant que processus complexe et dynamique, l'identité personnelle se constitue à l'articulation de trois axes principaux plus ou moins en tension. Tout d'abord, l'individu préserve le sentiment de rester le même au fil du temps et doit nécessairement s'adapter, en fonction de changements plus ou moins souhaités et/ou contrôlés : l'itinéraire professionnel intègre cet axe continuité / changement à travers un couplage entre histoire du sujet et changements du contexte professionnel. Par ailleurs, il élabore une image de soi en relation (accord, tension, contradiction) avec celles que, selon lui, les autres lui attribuent. Cela conduit à un sentiment de reconnaissance ou de non reconnaissance d'autrui essentiel dans la construction identitaire. Enfin, chacun fait en sorte de conserver une cohérence interne (unité) tout en développant de multiples facettes (diversité) sur lesquelles il jouer pour s'adapter à des situations changeantes (Roux-Perez, 2004 ; Tap, 1998).

L'identité professionnelle prend appui sur le "socle" de l'identité personnelle et y intègre des composantes professionnelles : pour trouver un équilibre, l'individu se représente la discipline, le métier, les pratiques professionnelles. En effet, envisagées comme des formes de connaissances porteuses de valeurs, les représentations sociales donnent sens à la pratique et légitiment une vision du monde (Jodelet, 1989 ; Moscovici, 1961). Elles orientent les conduites et amènent à privilégier certains types d'implication. Ainsi, l'individu s'engage d'une part en fonction des valeurs qui l'animent, d'autre part en fonction du sentiment de contrôle de la situation et des formes de reconnaissance sociale qui en découlent (Mias, 1998). L'implication au travail est donc révélatrice des rapports que le sujet établit avec son environnement professionnel. De ce point de vue, prendre en considération les modes d'implication privilégiés par les enseignants à l'intérieur ou à l'extérieur du système scolaire, c'est tenter de comprendre ce qui fait sens pour eux, donne une cohérence à leur action et fonde, au moins en partie, leur identité.

Dans cette perspective, représentations, valeurs et pratiques sont analysées pour rendre compte du rapport au métier (Charlot, 1997) ${ }^{1}$ et des ajustements permanents liés en partie à l'évolution de l'enseignement d'une discipline scolaire dans laquelle l'enseignant se reconnaît plus ou moins. Ainsi, la carrière des enseignants est envisagée dans sa dimension à la fois objective (succession des positions occupées par l'enseignant au cours de sa vie) et subjective c'est-à-dire en tant que processus de mise en cohérence par l'acteur de son cheminement professionnel (Hughes, 1937). L'intérêt porté aux étapes de la carrière intègre des moments clés, des points de bifurcation qui participent d'une redéfinition de soi en tant qu'acteur social. Le caractère « subi » ou « volontaire » du changement engage différemment le travail de recomposition de la personne vis-à-vis de la pluralité des mondes sociaux auxquels elle participe (Lahire, 1998).

L'étude présentée dans cette contribution se donne donc pour objet de comprendre la trajectoire d'un enseignant en repérant la manière dont il envisage son parcours professionnel, $y$ trouve des ancrages et développe des stratégies pour donner du sens au contexte de travail. Elle s'attache d'une part aux interactions entre histoire du sujet et contexte professionnel ; d'autre part elle s'intéresse aux modalités

\footnotetext{
${ }^{1}$ Pour Charlot (1997, p. 93) « le rapport au savoir est rapport au monde, à l'autre et à soi-même d'un sujet confronté à la nécessité d'apprendre ". Le rapport au savoir inclut les représentations, envisagées comme des systèmes de relation, d'interprétation, ancrées dans un réseau de significations. Il semble intéressant de transposer cette définition dans le cadre du rapport au métier.
} 
d'appropriation des dispositifs de formation et à leur impact sur d'éventuels processus de transformation en terme d'identité professionnelle (Barbier, 1996 ; Camilleri, 1990 ; Dubar, 1991 ; Lautier, 2001). Entre affirmation de soi et types de reconnaissance sociale ou institutionnelle, apparaissent des processus d'ajustement et de renégociation de l'identité. Déséquilibres, tensions, moments de crise sont dépassés si l'enseignant parvient à conserver la conscience de son unité, de sa continuité et une certaine maîtrise des choix qu'il peut opérer à un moment donné de sa trajectoire personnelle et professionnelle.

\section{Repères méthodologiques}

A la suite d'une enquête quantitative menée en 1998 portant sur un corpus de 381 enseignants, 12 entretiens ont été réalisés en 2000 parmi lesquels celui présenté dans cet article. Les données recueillies ont été traitées à partir d'une analyse structurale du discours (Demazière et Dubar, 1997) en repérant : a) les séquences ou épisodes du récit qui éclairent le parcours professionnel et en soulignent la dynamique ; b) les actants ou personnages qui interviennent dans le récit et avec lesquels s'établissent des systèmes de relations ; c) les propositions ou arguments destinés à convaincre l'interlocuteur, à défendre son point de vue (valeurs, identité pour soi et pour autrui, rapport à l'institution). Ces énoncés rendent compte des représentations à l'œuvre dans les discours. Ils sont retranscrits entre guillemets et en italique dans le texte. Par ailleurs, la démarche prend en compte la chronologie, les relations et les effets d'argumentation s'inscrivant dans une structure des oppositions révélatrice des tensions à dépasser pour rester équilibré dans un contexte professionnel particulier.

La contribution se centre sur le cas d'un professeur d'EPS, dénommé François dans l'étude. Le portrait proposé apparaît très révélateur de tensions entre ce qui constitue, du point de vue du sujet, une identité collective (fortement impulsée par l'action des inspecteurs sur le terrain et en formation continue) et ce qui constitue une identité singulière revendiquée. Choisi sur la base de ses réponses au questionnaire en 1998, cet enseignant défend une position démarquée vis-à-vis de l'évolution disciplinaire. Par ailleurs son engagement, à 50 ans, dans des pratiques compétitives en voile nécessitant de nombreux entraînements, le distingue de la plupart des enseignants d'EPS de son âge, optant le plus souvent pour des pratiques physiques de loisir.

\section{François : l'enseignant sportif humaniste}

\subsection{Un parcours en quête de reconnaissance}

Dès le début de l'entretien, qui se déroule deux ans après la passation du questionnaire, François met en avant la réussite de son fils, reçu brillamment aux épreuves du CAPEPS ${ }^{2}$. La filiation est donc assurée et cet enseignant apprécie de partager un métier pour lequel il se dit toujours motivé. Pourtant, des écarts existent : la réussite du fils est liée en partie à sa capacité à s'approprier une formation inscrite dans les nouvelles orientations institutionnelles et laissant une large part à la didactique. François ne se reconnaît pas dans cette approche et revient sur une scolarité difficile, largement compensée par de bons résultats sportifs : "mon identité d'enfant est passée par mes résultats sportifs. Si j'avais pas eu cette chose-là dans ma vie d'enfant, je ressemblerais à un certain nombre de mes élèves [en grande difficulté dans leur parcours scolaire] pour lesquels j'ai une certaine compassion quand même !»

A la suite de nombreux redoublements et face au sentiment d'être en échec, il choisit de suivre une formation courte ${ }^{3}$ pour accéder au métier d'enseignant. En rupture avec le projet familial quant à ce

\footnotetext{
${ }^{2}$ Le Certificat d'Aptitude au Professorat d'Education Physique et Sportive.

${ }^{3}$ Le diplôme de Maître d'Education Physique était présenté au terme de deux années d'étude après l'obtention du Brevet en fin de scolarité du collège
} 
choix professionnel jugé dévalorisant, il poursuit et réussit dans une formation fondée sur une expertise sportive et pédagogique très éloignée des discours didactiques actuels. L'entrée dans le métier est donc vécue comme un paradoxe : il est nommé dans l'établissement où deux ans auparavant, il était un élève en échec. Plusieurs expériences ponctuent son parcours professionnel et sont évoquées comme ayant participé d'une évolution : en tant que maître-nageur, il a pu développer des qualités d'écoute et une individualisation des apprentissages ; son engagement au sein de la fédération de Tennis lui a valu une reconnaissance pour sa réflexion sur de nouvelles approches pédagogiques. Cet ensemble d'éléments lui permet de construire une identité professionnelle reconnue de tous jusqu'au moment où l'intégration de l'EPS au sein de l'Education nationale invite à questionner les grands enjeux éducatifs, à se préoccuper des savoirs fondamentaux pour favoriser une culture corporelle «ouverte ». En conséquence, il devient nécessaire de proposer aux élèves des expériences diversifiées prenant appui sur des APSA $^{4}$ complémentaires pour lesquelles François n'a pas été formé. Les attentes de l'institution envers les enseignants, notamment en terme de formation continue, deviennent plus pressantes. La réflexion didactique opère alors comme une rupture, renforcée par les positions et l'action d'un inspecteur pédagogique très critique vis-à-vis de certaines pratiques professionnelles dans lesquelles François se reconnaît. Cet écart l'amène à revendiquer une identité singulière, fondée sur des valeurs, des représentations professionnelles en lien avec des pratiques et des choix assumés.

De ce point de vue, son parcours professionnel est assez caractéristique d'un besoin de continuité : enseignant depuis 30 ans dans l'établissement où il était élève, il envisage d'y rester pour terminer sa carrière, sans chercher à améliorer son statut, car son rapport aux savoirs scolaires reste si douloureux qu'il n'a jamais envisagé de tenter un concours interne : «je peux pas aller me livrer comme ça... J'en suis psychologiquement pas capable!»

\subsection{Des valeurs construites autour des pratiques partagées}

Les valeurs que défend François semblent liées à son histoire scolaire. Attentif aux élèves en difficulté dont il se fait le porte-parole, soucieux de la qualité des relations humaines, il défend aussi l'idée que, par l'EPS, il peut contribuer à «faire sortir les jeunes d'une certaine vacuité physique et mentale », leur donner les moyens de se prendre en charge, de croire en leur potentiel.

Il situe l'utilité de son action au plan éducatif à partir de deux dimensions : 1 'amour des élèves et le développement de leurs capacités physiques. Ainsi, il opère sans cesse des va-et-vient entre expériences corporelles et incidences sur le plan de la construction de la personne, sans pour autant en expliciter les mises en œuvre. Se pencher sur les procédures pédagogiques et didactiques inhérentes à l'acte d'enseigner, relève pour cet enseignant d'une forme de conceptualisation, trop éloignée du "faire" auquel il reste attaché par dessus tout.

Ainsi, au fil du discours, le groupe professionnel est présenté de façon clivée : d'un côté les «concepteurs», de l'autre les «praticiens», d'un côté les «jeunes », de l'autre les «anciens». Ces derniers, auxquels il s'identifie, sont décrits comme des "artisans" : inscrits dans le faire, ils peuvent « devenir des locomotives pour les jeunes ». On repère, à travers ces clivages une tension entre théorie et pratique. Pourtant, pour cet enseignant, la recherche en éducation physique est fondamentale ; mais celle-ci, basée sur la confiance et le respect, doit se faire dans la continuité : "on ne peut pas d'une chiquenaude, repousser vingt ans ou vingt-cinq ans d'expérience et de pratique sur le terrain. On peut pas balancer comme ça les choses ».

\footnotetext{
${ }^{4}$ Activités Physiques Sportives et Artistiques : dans les programmes officiels d'EPS, à partir de 1996, huit groupes d'activités doivent être proposés aux élèves dans leur scolarité, pour permettre une éducation physique complète et équilibrée : sports collectifs, sports de raquettes, activités athlétiques, gymniques, aquatiques, artistiques, de combat, de pleine nature.
} 
ROUX-PEREZ.T, MCF, IUFM des Pays de la Loire, Laboratoire du CREN, Nantes, Identité professionnelle des enseignants : entre singularité des parcours et modes d'ajustement aux changements institutionnels. Revue Savoirs, 2006, n¹1, pp. 107-123.

\subsection{Un métier appréhendé de façon singulière}

Les représentations sur le métier sont assez démarquées de celles qui dominent à l'intérieur du groupe professionnel (Roux-Perez, 2003 b). François associe le métier d'enseignant à celui d'éducateur pris au sens d'élever un enfant: "l'être humain va s'élever, va devenir un bel être humain qui va développer plein de choses ». Il évoque aussi celui de technicien, capable de démontrer des savoir-faire aux élèves et de corriger leurs erreurs. Enfin, l'image du bricoleur est proposée au filtre de ses propres compétences : «je crois que j'enseigne ce que je suis donc, je bricole beaucoup. Au sens très large du terme, je suis un bricoleur de l'éducation ». Ainsi, le système "éducateur-technicien-bricoleur" permet à François de donner du sens à son action professionnelle en la référant à une histoire singulière.

Complémentairement, il privilégie certaines facettes du métier d'enseignant ; pour lui, un enseignant d'EPS, c'est avant tout une personne qui "sait s'adapter au contexte et se centrer sur l'essentiel" c'est-àdire sur les interactions développées au travers de la discipline : «j'ai toujours envie de dépasser cette discipline. Faire en sorte que les gens se respectent, qu'il dédramatisent un certain nombre de choses qui sont pas importantes». Ainsi, l'enseignant "connaît la psychologie des adolescents », élément essentiel pour pouvoir s'adapter aux élèves, en lien avec la nécessaire connaissance de soi. Enfin, il «maîtrise bien les APSA », argument récurrent dans le discours et utilisé comme une évidence.

\subsection{Entre définitions de soi et regard d'autrui}

François se définit comme un enseignant organisé autour de l'action, "en prise directe avec la réalité ». Par ailleurs, il insiste sur la nécessité d'enseigner de façon authentique et se caractérise comme un «homme de passion ». Cette dimension se retrouve dans la pratique de voile, inscrite dans un réseau relationnel et lui permettant de rester actif : "à la limite, la compétition m'intéresse pas tellement... c'est surtout le fait que je vais pas enfiler mes charentaises ». Cet engagement est en lien avec une certaine peur de vieillir, de ne plus être à la hauteur : «dans mon job, quand je vois les générations qui passent... j'espère ne pas être un ancien combattant». Ayant construit une identité autour des pratiques sportives dans lesquelles il était performant, reconnu socialement à travers elles, il ne peut envisager sans crainte la diminution de ses moyens physiques et un avenir plus tranquille dans lequel, pour l'instant, il lui est impossible de se projeter. Enfin, François se définit comme un enseignant «humaniste », préoccupé avant tout par la relation à l'autre : "je suis quelqu'un d'intimiste, ce qui fait que je suis assez centré sur l'élève, l'élève en tant qu'individu».

Le regard supposé porté par les autres vient en écho à cette définition de soi. Si un changement notable est évoqué dans le regard familial vis-à-vis d'un métier au départ dévalorisé, François se sent reconnu depuis toujours par ses collègues de travail pour ses nombreux savoir-faire. Dans l'établissement, en revanche, il passe "pour un enseignant qui dérange », notamment dans ses prises de position lors des conseils de classe. S'insurgeant sur les conclusions hâtives de certains professeurs à partir des seuls résultats scolaires, il fait donc encore, trente ans après et par élève interposé, le procès d'un système qui n'a pas voulu de lui.

Enfin, du point de vue des élèves, après avoir eu une image de sportif polyvalent, il se dit perçu comme une sorte de penseur, un sage : «les élèves me voient aussi un peu atypique. Ils me disent : pourquoi vous êtes pas prof de philo?».

\subsection{Un rapport ambivalent à l'institution : entre adhésion et résistances}

S'inscrivant dans un rapport ambivalent à l'institution, il réinterprète les textes officiels prenant appui sur ce qui lui semble une réelle avancée disciplinaire : l'évaluation sous forme de contrôle continu et la prise en compte des progrès sont considérés comme l'occasion de motiver les élèves, de les obliger à 
s'investir et à travailler. Mais transformer les modalités d'évaluation n'évite pas une réflexion sur les contenus et les procédures à mettre en œuvre pour que l'élève soit acteur de ses apprentissages. A ce niveau, François refuse de passer d'une évaluation globale à une évaluation critériée donnant des repères à l'élève. Il considère par ailleurs la pédagogie du projet comme une dérive démagogique et les pratiques en mixité comme impossibles tant les écarts filles -garçons lui apparaissent importants dans les activités qu'il propose.

Par ailleurs, il fustige l'inspecteur qui n'a pas su s'appuyer sur « l'expertise des anciens ». Ainsi, ne se sentant pas reconnu, François prend ses distances et critique un cadre d'inspection élaboré à partir de trois thèmes majeurs : construire des contenus et des modes d'évaluation adaptés aux élèves et en lien avec les programmes, conduire les leçons de manière à organiser les apprentissages et enfin favoriser les progrès de tous les élèves. Cet ensemble d'exigences lui pose problème et rend l'inspection « tout à fait inadaptée et formelle » dans la mesure où elle n'intègre pas une valeur fondamentale sur laquelle il revient sans cesse : le respect des personnes.

Enfin, l'EPS reste pour François une discipline à part, en avance sur les autres à condition qu'elle ne se détourne pas de sa mission première : le développement des conduites motrices par la pratique sportive. Or, il reproche à l'évolution disciplinaire une dérive intellectuelle ne profitant qu'aux élèves déjà en réussite scolaire. A travers l'interprétation des discours de l'inspecteur qui le déstabilisent, François se sent humilié et met en place des modes de résistance pour justifier ses pratiques. Tout d'abord, il se définit à travers l'expertise qu'il a développée dans des activités sportives qu'il juge fondamentales. Critique envers certains enseignants qui utilisent d'autres activités mais qu'il estime incompétents, il énonce à plusieurs reprises qu'en pédagogie : «il vaut mieux ne pas faire plutôt que mal faire ». Il souligne par ailleurs la nécessité d'une démarche inductive dans l'innovation : «un concept, quel qu'il soit, peut être indiscutable dans sa théorie et peut être complètement inopérant sur le terrain ».

Enfin, pour François, les espaces de reconnaissance sont construits ailleurs, en dehors du système scolaire : au niveau fédéral (il est entraîneur) et dans le cadre de pratiques compétitives (voile) où il est en réussite. Il trouve donc des formes d'implication à l'extérieur du système scolaire, mettant à distance toute perte de sens dans l'avancée disciplinaire et se préservant d'une perte de soi dans un système qui ne le reconnaît plus. Pourtant, au-delà des résistances, il doit trouver le moyen de construire un monde cohérent.

La partie suivante montre les stratégies mises en œuvre pour résoudre les tensions entre continuité et changement, entre image de soi pour soi et de soi pour autrui. Elle met en relief la manière dont cet enseignant dépasse les clivages en se situant au niveau des valeurs.

\section{Discussion}

\subsection{La construction d'un monde cohérent}

Pour rester en cohérence avec lui-même, construire en quelque sorte un « monde qui tient » (Derouet, 1992) intégrer les événements, situations, interactions qui le conduisent à questionner ses propres ancrages, François opère de multiples transactions avec son environnement institutionnel et humain.

\subsubsection{Résoudre les tensions entre continuité et changement}

François fonde son identité d'enseignant sur un vécu d'élève en échec scolaire et en réussite par le sport. Il organise son système de valeurs autour des pratiques sportives et de l'attention particulière aux

\footnotetext{
${ }^{5}$ Le terme de monde est envisagé ici comme une totalité d'appartenance, désignant un ensemble culturel spécifique porteur de significations. Il exprime donc un mode de compréhension unificateur, un noyau de significations commun (Encyclopédia Universalis).
} 
élèves en difficulté. Le retour dans l'établissement de sa ville natale où il était en échec scolaire mais très reconnu pour ses performances sportives, est un signe de forte continuité, même s'il a changé depuis de statut et d'image.

En même temps, son implication externe au système scolaire s'inscrit dans la continuité. Elle lui donne l'impression, à cinquante ans, de pouvoir toujours se confronter à l'effort et de conserver un corps relativement performant. C'est l'occasion pour lui de garder ses ancrages. En effet, en début de carrière, François donne l'image d'un enseignant polyvalent, impressionnant les élèves par ses compétences sportives. A cinquante ans, des problèmes physiques apparaissent, le conduisant à changer quelque peu son mode d'enseignement. Il développe un discours sur l'EPS, sur les valeurs de l'effort, sur le comportement de l'individu confronté aux pratiques de pleine nature. Il privilégie les valeurs humaines, incontournables dans ce métier et en écho à sa propre histoire.

En fait, le changement le plus important vient des recommandations institutionnelles avec lesquelles François se sent en décalage. Pourtant, il revendique une implication dans la réflexion pédagogique engagée avec la fédération de tennis ; en continuité avec le sport envisagé dans sa dimension éducative, cette réflexion aurait du pouvoir enrichir celle menée sur la didactique de l'EPS : «j'ai pu voir les apprentissages en tennis par l'autre bout de la lorgnette... Ils m'ont reposé la question de ma façon d'enseigner au lycée. Et j'ai pu rebondir là-dessus. Ces choses-là m'ont fait bouger dans les autres pratiques ». Par ailleurs, ancré dans son établissement, il reste prêt à saisir les opportunités qui adviennent à l'occasion de rencontres avec des enseignants plus jeunes : un échange de savoirs réalisé sur le tas, fait de tours de mains, de "choses qui marchent", loin des formations devenues trop conceptuelles à ses yeux.

C'est en voyant faire les autres (son fils, une jeune enseignante de l'équipe d'EPS) que François se questionne. La formation continue devrait l'aider à changer ses pratiques, à condition qu'elle relie compétences des anciens et des nouveaux enseignants. Tout au long de l'entretien, il élabore un système d'opposition entre la formation de son fils et la sienne, qui confirme ses représentations sur le métier. Pour préserver à tout prix une continuité entre père et fils, cet enseignant défend l'idée d'une formation respectueuse des différences, articulant réflexion didactique et mises en œuvre pédagogiques. Si cette recherche d'articulation existe, il ne la perçoit pas. Les raisons sont sans aucun doute liées à un sentiment de non-reconnaissance et à un écart jugé trop grand entre sa formation initiale et les recommandations institutionnelles. En même temps, François pointe son retrait vis-à-vis de la formation continue : "je dois être un peu paresseux aussi. Donc, il y a des moments ou j'ai laissé courir le train ». Ses choix d'implication à l'extérieur du système scolaire ont pu aussi lui permettre de sauvegarder des compétences qu'il n'avait pas réellement envie de transformer.

\subsubsection{Gérer les écarts entre définition de soi et regard d'autrui}

François a toujours été reconnu dans le milieu fédéral. Athlète accompli, en réussite comme entraîneur de tennis autodidacte, valorisé en tant qu'expert de la pédagogie au sein de la fédération, actuellement compétiteur en voile, il a pu développer une image positive de lui-même, dans des registres de compétences différents, caractéristiques d'une polyvalence sportive. Cette diversité d'expériences vécues en dehors du monde scolaire, lui a permis de construire une identité professionnelle, fondée sur la connaissance des APSA et les démonstrations qui l'accompagnent, si nécessaire. François s'exprime dans le «faire». Pour lui l'institution, par le biais de l'inspecteur, n'a pas su reconnaître ses compétences. Face à cela, il énonce des représentations professionnelles lui permettant de rester équilibré en conservant un ancrage sur les pratiques et non sur l'élève acteur de ses apprentissages. De ce point de vue, la mixité est impossible à mettre en œuvre, le projet de l'élève ne fait pas sens et les critères d'évaluation sont peu opérants; le tout appartient au monde des discours (le « dire »).

Bien qu'il se dise centré sur l'élève et souhaite dépasser le seul cadre de la discipline, François ne s'implique pas dans l'établissement malgré les sollicitations dont il semble être l'objet, et préfère passer 
pour un «empêcheur de tourner en rond», défenseur de l'opprimé, refusant de mettre des notes au premier trimestre et revendiquant une dimension humaine dans son enseignement. Le seul regard qui l'interpelle semble être celui de son fils : une vision du métier différente qui le renvoie à des insuffisances qu'il faut assumer, y compris dans ce qu'il revendique comme son domaine de compétences : le savoir-faire pédagogique. A ce sujet, François souligne certaines difficultés à changer ses pratiques : «il y a des moments où je suis pas très fier de moi, quoi, dans mon boulot... parce que là on parle, mais faudrait voir aussi la réalité de terrain. Elle est pas toujours si terrible que ça!» Pour gérer ces tensions, il s'en réfère à l'inspecteur qui, de son point de vue, "a loupé le coche...a raté le train" et n'a pas su s'appuyer sur les atouts des uns et des autres. L'image de soi pour soi est sauve et François garde ses compétences, décalées avec les attentes institutionnelles mais reliées à son histoire et en phase avec ses propres valeurs.

\subsubsection{Dépasser les clivages en se situant au niveau de valeurs}

La recherche de mise en cohérence des différents modes d'implication, à l'extérieur ou à l'intérieur du système scolaire, s'opère autour de la valorisation de l'action, pour soi comme dans la façon d'appréhender l'EPS. De la même manière, l'engagement fédéral trouve un écho possible: les innovations pédagogiques importées de l'extérieur changent le regard sur les pratiques des élèves. L'enseignant expert peut favoriser les apprentissages des élèves et les amener à progresser.

Enfin, pour dépasser un rapport aux savoirs scolaires, douloureux pour le père et valorisant pour le fils, pour gérer les différences de conception du métier liées aux contenus de la formation initiale et aux expériences singulières, François se situe sur le plan des valeurs humaines. Au-delà de leurs compétences professionnelles respectives, son fils doit être avant tout un homme juste qui «aime tous ses élèves », particulièrement ceux en échec. La recommandation ultime porte la trace de cette valeur suprême : «je lui dis souvent : ce qui m'intéresse, c'est que tu sois un bel être humain ».

Symboliquement, ce discours conforte le lien entre eux et atténue d'éventuels clivages conceptuels. Tous deux sont face à des jeunes en devenir, qu'il faut accompagner. Ceci suppose de dépasser le cadre strict de la discipline. Pourtant, François ne s'inscrit dans aucun projet particulier au sein de l'établissement et refuse d'être professeur principal. C'est donc en terme de prise en compte du comportement, de soutien psychologique non institutionnel auprès d'élèves en difficulté qu'il faut entendre son engagement. Un engagement en lien avec son histoire et cette image "de malheureux petit canard gris d'un système scolaire donné ».

\subsection{Apports de l'étude à la formation des enseignants}

Au-delà de cette étude de cas, les résultats de l'enquête mettent en relief la diversité avec laquelle chaque enseignant, porteur d'une histoire singulière, investit son parcours et se saisit des opportunités professionnelles ou personnelles qu'il rencontre. L'entrée dans le métier joue un rôle déterminant par rapport à l'engagement dans une carrière. Dans le cas de François, il s'agit d'une revanche : devenir enseignant dans un système où l'on a été un élève en échec donne l'occasion de défendre ceux dont les difficultés semblent identiques et conduit à rejouer sa propre histoire sur la scène scolaire.

Par ailleurs, l'analyse permet de repérer le poids du parcours scolaire, de la formation initiale et de l'histoire sportive sur le rapport à la formation. Un souvenir douloureux de l'école, associé à un sentiment d'échec, bloque François dans sa trajectoire professionnelle. Les perspectives de progression sont saisies ailleurs, dans l'espace sportif, plus facile d'accès mais renforçant un clivage théoriepratique qui fonde le système de justification à propos des modes d'implication privilégiés. S'impliquer au niveau fédéral, c'est valoriser les pratiques et se trouver en réussite ; le faire au niveau de la formation continue, c'est courir le risque d'être déstabilisé, voire dépassé. François choisit donc de se 
former «sur le tas», en fonction des opportunités et des rencontres qu'il peut faire dans l'établissement.

De façon plus générale, cette étude éclaire les questions liées à la formation des enseignants, dans la mesure où celle-ci se heurte souvent à la diversité des publics, de leurs caractéristiques et de leurs attentes. De ce point de vue, l'institution devrait offrir aux enseignants les moyens de réfléchir à la qualité des processus enseignement/apprentissage dans des contextes de travail particuliers. Cela suppose, en formation, d'apprendre à s'observer mutuellement de façon étayée, à évoquer réussites et difficultés, à s'enrichir des complémentarités des uns ou des autres. Pour éviter que certains enseignants ne délaissent une formation qui, de leur point de vue, ne les prend plus suffisamment en compte, il semble indispensable de créer, dans des espaces de travail diversifiés, reconnaissance mutuelle de compétences et échange de savoirs. C'est à ce prix que pourraient être réduits certains écarts préjudiciables pour la dynamique des équipes pédagogiques. Ainsi, privilégier en formation un axe pratique-théorie-pratique conduirait sans doute à renforcer, pour les enseignants ancrés dans l'action (et les pratiques sportives), une appropriation progressive de la réflexion didactique et des procédures centrées sur les apprentissages des élèves. On peut faire l'hypothèse de transformations potentielles des pratiques pédagogiques des enseignants, si ces derniers se sentent respectés dans leur culture et plus largement dans leur histoire. Dominice rend largement compte de ce phénomène en indiquant que l'offre de formation doit inscrire les apports de connaissances externes dans un processus d'évolution personnelle et réhabiliter des savoirs ouverts à l'altérité, à l'interculturel, à l'intergénérationnel. " $L a$ volonté des transmissions intergénérationnelles participe de ce mouvement d'ancrage de l'apprenant dans son historicité » $(2004,60)$.

\section{Conclusion}

Au terme de ce travail, on saisit combien les trajectoires professionnelles s'organisent entre sentiment de compétence et espaces de reconnaissance sociale investis. Ces derniers participent de la construction identitaire et invitent l'individu à s'engager davantage dans un secteur où il peut construire des repères et donner sens à son action.

Au-delà des arrangements entre soi et le contexte, l'étude révèle les processus en jeu dans la construction de l'identité professionnelle au travers de représentations permettant aux enseignants d'EPS de fonder et de rationaliser leurs choix professionnels. Elle met en relief des cohérences provisoires, laissant place à d'éventuels ajustements, lorsque surviennent des changements institutionnels, humains ou contextuels dans le parcours des enseignants. En réponse à ces changements plus ou moins souhaités, ces derniers aménagent et recomposent certains éléments constitutifs de leur identité en fonction des situations, pour s'adapter et construire un monde cohérent entre contraintes de l'environnement (institutionnel et humain) et diversité des ressources de chacun. Ces ressources, puisées dans les expériences passées ou présentes, s'opérationnalisent dans la pluralité des mondes investis par le sujet et sont constitutives d'une identité revendiquée. Orientation des actions et formes d'engagement sont alors justifiées par des représentations et des valeurs fondatrices de choix professionnels ou personnels. La prise en compte de ces représentations semble indispensable pour donner sens à la formation et engager les enseignants dans les processus de transformation attendus par l'institution. 
ROUX-PEREZ.T, MCF, IUFM des Pays de la Loire, Laboratoire du CREN, Nantes, Identité professionnelle des enseignants : entre singularité des parcours et modes d'ajustement aux changements institutionnels. Revue Savoirs, 2006, n¹1, pp. 107-123.

\section{Bibliographie}

Barbier, J.-M. (1996). De l'usage de la notion d'identité en recherche. Education permanente, 128, 11-26.

Camilleri, C. (Sd) (1990). Stratégies identitaires. Paris : PUF.

Charlot, B. (1997). Du rapport au savoir. Paris : Anthropos.

Demazière, D. \& Dubar, C. (1997). Analyser les entretiens biographiques. Paris : Nathan.

Derouet, J.-L. (1992). Ecole et justice. Paris : Métailié.

Dominice, P. (2004). Le désarroi adulte face au savoir. Savoirs, 4, 53-62.

Dubar, C. (1991). La socialisation : construction des identités sociales et professionnelles. Paris : Armand Colin.

Dubar, C. (2000). La crise des identités, l'interprétation d'une mutation. Paris : PUF.

Dubet, F. (2002). Le déclin de l'institution. Paris : La découverte.

Hughes, E.-C. (1937). Institutional office and the person. American Journal of Sociology, 43, 3, 404-413.

Jodelet, D. (1989). Les représentations sociales. Paris : PUF.

Lahire, B. (1998). L'homme pluriel. Paris : Nathan.

Lautier, N. (2001). Psychosociologie de l'éducation. Paris : Armand Colin.

Merton, R. (1957). Social Théory and Social Structure. New York : The Free Press.

Mias, C. (1998). L'implication professionnelle dans le travail social. Paris : L'Harmattan.

Moscovici, S. (1961). La psychanalyse, son image et son public. Paris : PUF.

Roux-Perez, T. (2003a). Identité professionnelle et modes d'implication privilégiés chez les enseignants d'EPS. Les Sciences de l'éducation, pour l'Ere nouvelle, vol 36, 4, 37-68

Roux-Perez, T. (2003b). L'identité professionnelle des enseignants d'EPS : entre valeurs partagées et interprétations singulières. STAPS, vol 25, 63, 75-88.

Roux-Perez T. (2004). Stratégies d'adaptation des enseignants d'EPS et recompositions identitaires. In M. Loquet \& Y.Léziart, Cultures sportives et artistiques. Formalisation des savoirs professionnels. Rennes : UFRAPS Université Rennes 2 / ARIS, 239-242.

Tap P. (1998). Marquer sa différence. In : J.C. Ruano-Borbalan. (Sd). L'identité : l'individu, le groupe, la société. Auxerre : Sciences Humaines, 65-68. 